\title{
Geometric Alignments of the Subgrid-Scale Force in the Atmospheric Boundary Layer
}

\author{
Chad W. Higgins - Charles Meneveau • \\ Marc B. Parlange
}

Received: 25 June 2008 / Accepted: 7 April 2009 / Published online: 9 May 2009

(C) Springer Science+Business Media B.V. 2009

\begin{abstract}
In recent years field experiments have been undertaken in the lower atmosphere to perform a priori tests of subgrid-scale (SGS) models for large-eddy simulations (LES). The experimental arrangements and data collected have facilitated studies of variables such as the filtered strain rate, SGS stress and dissipation, and the eddy viscosity coefficient. However, the experimental set-ups did not permit analysis of the divergence of the SGS stress (the SGS force vector), which is the term that enters directly in the LES momentum balance equations. Data from a field experiment (SGS2002) in the west desert of Utah, allows the calculation of the SGS force due to the unique $4 \times 4$ sonic anemometer array. The vector alignment of the SGS force is investigated under a range of atmospheric stabilities. The eddy viscosity model is likely aligned with the measured SGS force under near-neutral and unstable conditions, while its performance is unsatisfactory under stable conditions.
\end{abstract}

Keywords Atmospheric boundary layer - Large-eddy simulation · Smagorinsky model · Statistical geometry $\cdot$ Subgrid scale $\cdot$ Vector model

\section{Introduction}

Large-eddy simulation (LES) is a numerical technique to simulate turbulent geophysical flows in which large-scale motions with a characteristic size larger than the filter size $\Delta$

C. W. Higgins $(\varangle) \cdot$ M. B. Parlange

School of Architecture, Civil and Environmental Engineering,

Ecole Polytechnique Federale de Lausanne, Lausanne, Switzerland

e-mail: chad.higgins@epfl.ch

C. Meneveau

Center for Environmental and Applied Fluid Mechanics, Johns Hopkins University,

Baltimore, MD, USA

C. Meneveau

Department of Mechanical Engineering, Johns Hopkins University, Baltimore, MD, USA 
are resolved, while motions with a characteristic size smaller than $\Delta$ (subgrid scales, SGS) must be parameterized. The LES equations for momentum transport are obtained by spatially filtering the Navier-Stokes and continuity equations, giving

$$
\begin{aligned}
\frac{\partial \tilde{u}_{i}}{\partial t}+\tilde{u}_{j} \frac{\partial \tilde{u}_{i}}{\partial x_{j}} & =-\frac{1}{\rho} \frac{\partial \tilde{p}}{\partial x_{i}}+\frac{\partial \tau_{i j}}{\partial x_{j}}+f_{i}, \\
\frac{\partial \tilde{u}_{i}}{\partial x_{i}} & =0
\end{aligned}
$$

where $\sim$ represents spatial filtering, $u_{i}$ is the velocity, $p$ is the pressure, $\rho$ is the density, $t$ is time, $x_{i}$ is the spatial coordinate, $f_{i}$ represents additional forcing that could include buoyancy, mean pressure gradients, etc, and $\tau_{i j}=\widetilde{u_{i} u_{j}}-\tilde{u}_{i} \tilde{u}_{j}$ is the SGS stress and must be parameterized. In what follows, for simplicity and without loss of generality, we replace $\tau_{i j}$ with its trace-free part $\tau_{i j}-\delta_{i j} \tau_{k k} / 3$.

The SGS stress enters the LES equations (Eqs. 1a, b) through a divergence term $\partial \tau_{i j} / \partial x_{j}$. The SGS force has previously been investigated using direct numerical simulation (DNS) by Clark et al. (1979), who found that correlations between individual components of the SGS stress and the stress calculated from the nonlinear model were essentially the same at the tensor $\left(\tau_{i j}^{\text {measured }}\right.$ and $\left.\tau_{i j}^{\text {modeled }}\right)$ and vector $\left(\partial_{j} \tau_{i j}^{\text {measured }}\right.$ and $\left.\partial_{j} \tau_{i j}^{\text {modeled }}\right)$ levels. They also observed that the correlations between the SGS stress and the stress calculated using the Smagorinsky model improved at the vector level. The SGS force has not yet been studied in the context of atmospheric surface-layer turbulence. Previous experiments (Porté-Agel et al. 1998, 2000, 2001a; Tong et al. 1998; Horst et al. 2004; Kleissl et al. 2004) lacked a sufficient number of spatial sampling points in the vertical direction to fully resolve the SGS force components.

An experiment (Higgins et al. 2007) was explicitly designed to provide such additional sampling points to enable the study of the SGS force within the atmospheric surface layer. In this paper, we present analysis of these data to study the geometric properties of the SGS force and its parameterization by eddy viscosity and nonlinear models in the atmospheric surface layer. Briefly, these parameterizations are the well-known closure schemes for the SGS stress that include the Smagorinsky (1963), eddy viscosity, model,

$$
\tau_{i j}^{\text {Smag }}=-\left(c_{s} \Delta\right)^{2}|\tilde{\mathbf{S}}| \tilde{S}_{i j},
$$

as well as the nonlinear model (Clark et al. 1979; Liu et al. 1994; Borue and Orszag 1988; Meneveau and Katz 2000),

$$
\tau_{i j}^{n l}=C_{n l} \Delta^{2}\left[\frac{\partial \tilde{u}_{i}}{\partial x_{k}} \frac{\partial \tilde{u}_{j}}{\partial x_{k}}-\delta_{i j} \frac{1}{3} \frac{\partial \tilde{u}_{m}}{\partial x_{k}} \frac{\partial \tilde{u}_{m}}{\partial x_{k}}\right] .
$$

In the above equations, $\tilde{S}_{i j}$ is the filtered strain rate, $|\tilde{\mathbf{S}}|$ is the strain rate magnitude, $c_{s}$ is the Smagorinsky model coefficient, and $C_{n l}$ is the nonlinear model coefficient. The present study makes use of data collected using a unique $4 \times 4$ sonic anemometer array (Higgins et al. 2007) and generalizes the analysis technique developed earlier in Higgins et al. (2004) in a study of the SGS heat flux vector.

\section{Field Experiment and Analysis}

Sixteen sonic anemometers were deployed at the Surface Layer Turbulence and Environmental Science Test (SLTEST) site in the western desert of Utah on July 8-10, 2002; the upwind 
fetch was $\mathrm{O}(100 \mathrm{~km})$. The anemometers were arranged in a 4 by 4 grid orthogonal to the mean wind direction, with the array centre $4.5 \mathrm{~m}$ above the ground, with horizontal and vertical spacings between the anemometers of $1 \mathrm{~m}$. Using $2 \times 2$ sonic anemometers for the spatial filtering, a filter size $(\Delta)$ of $2 \mathrm{~m}$ can be achieved. A photograph of the experimental set-up is given in Fig. 1. Measurements of the temperature and of the three components of the velocity vector were logged at $20 \mathrm{~Hz}$ for each anemometer simultaneously. The parameter $z / L$ was used to classify the data into three stability regimes, where $z$ is taken as the reference sonic height $(5 \mathrm{~m}), L$ is the Obukhov length, $L=-u_{*}^{3} T_{a} \rho c_{p} / \kappa g H, u_{*}=\left(\left\langle u^{\prime} w^{\prime}\right\rangle^{2}+\left\langle v^{\prime} w^{\prime}\right\rangle^{2}\right)^{\frac{1}{4}}$ is the friction velocity, $H=\rho c_{p}\left\langle w^{\prime} T^{\prime}\right\rangle$ is the sensible heat flux, $\rho$ is the density of air, $c_{p}$ is the specific heat of air, $T_{a}$ is the reference air temperature, $\mathrm{g}$ is the acceleration of gravity, and $\kappa=0.4$ is von Kármán's constant. In this study we define data obtained with a stability criterion of $|z / L|<0.015$ to be near neutral, $z / L>0.08$ to be stable, and $z / L<-0.1$ to be unstable. The total range of $z / L$ varied from -5 to 2.5 over the course of the experiment. Each time segment analyzed is approximately $30 \mathrm{~min}$, and in total there were 43 unstable segments, 30 near-neutral segments and 5 stable segments. Three-dimensional filtering is done at a scale of $\Delta=2 \mathrm{~m}$ in all three dimensions by applying a box filter in the vertical and cross-stream horizontal directions and a Gaussian filter in the stream-wise (temporal) direction. Derivatives in all directions are calculated with second-order finite difference schemes using a staggered grid in the $y-z$ plane. Finally, linear interpolation is used to express all derived quantities at the array centre.

As a first step, we evaluated the correlation coefficients between individual stress and force components, to compare with the original results of Clark et al. (1979). We find correlations between the SGS stress and filtered strain rate components in the range of 0.1 to 0.3 , while correlation coefficients between the SGS force components and the divergence of the strain are in a range from 0.2 to 0.5 . The trends are similar to those found by Clark et al. for isotropic turbulence.

The main objective of the present paper is to examine these issues from a more geometric viewpoint, and so we focus on angles between the vectors. While correlation coefficient and geometric alignment are somewhat related, they do not map one-to-one with each other since the alignments scale out the vector magnitudes that are "mixed in" for the case of the correlation coefficients. We calculate the cosine of the angle between the measured and modelled quantities to ascertain the relative orientation of the SGS force and its parameterizations. Probability density functions (PDFs) of $\cos \theta=\frac{\partial_{j} \boldsymbol{\tau}_{i j} \partial_{k} \boldsymbol{\tau}_{i k}^{\text {mod }}}{\left|\partial_{n} \boldsymbol{\tau}_{m n}\right|\left|\partial_{l} \tau_{h l}^{\text {mod }}\right|}$ are computed and presented for the neutrally buoyant atmosphere in Fig. 2. There is strong alignment between the measured SGS force and the divergence of $\boldsymbol{\tau}_{i j}$ obtained from the nonlinear model $\left(\nabla \cdot \tau^{n l}\right)$ as indicated by the increased probability at $\cos \theta=1$ (solid line). The alignment of the measured SGS force with the divergence of $\boldsymbol{\tau}_{i j}$ obtained from the Smagorinsky model ( $\nabla \cdot \boldsymbol{\tau}^{\text {Smag }}$ dashed line) also has an increased probability at $\cos \theta=1$. A second weaker mode of anti-alignment $\cos \theta=-1$ is observed.

Figure 3 presents the probability density functions of $\cos \theta$ conditionally sampled based on the magnitude of the SGS force. For high magnitudes of the SGS force, $|\nabla \cdot \tau|>$ $0.15 \mathrm{~m}^{2} \mathrm{~s}^{-3}$, we observe that there are increases in the probability of co-alignment of the SGS force with both the divergence of the SGS stress with Smagorinsky and the nonlinear model. This indicates that the models perform better as the SGS force magnitude increases.

To investigate possible implications on the energy cascade and SGS energy flux between large and small scales, the two dimensional joint probability density function (JPDF) of 


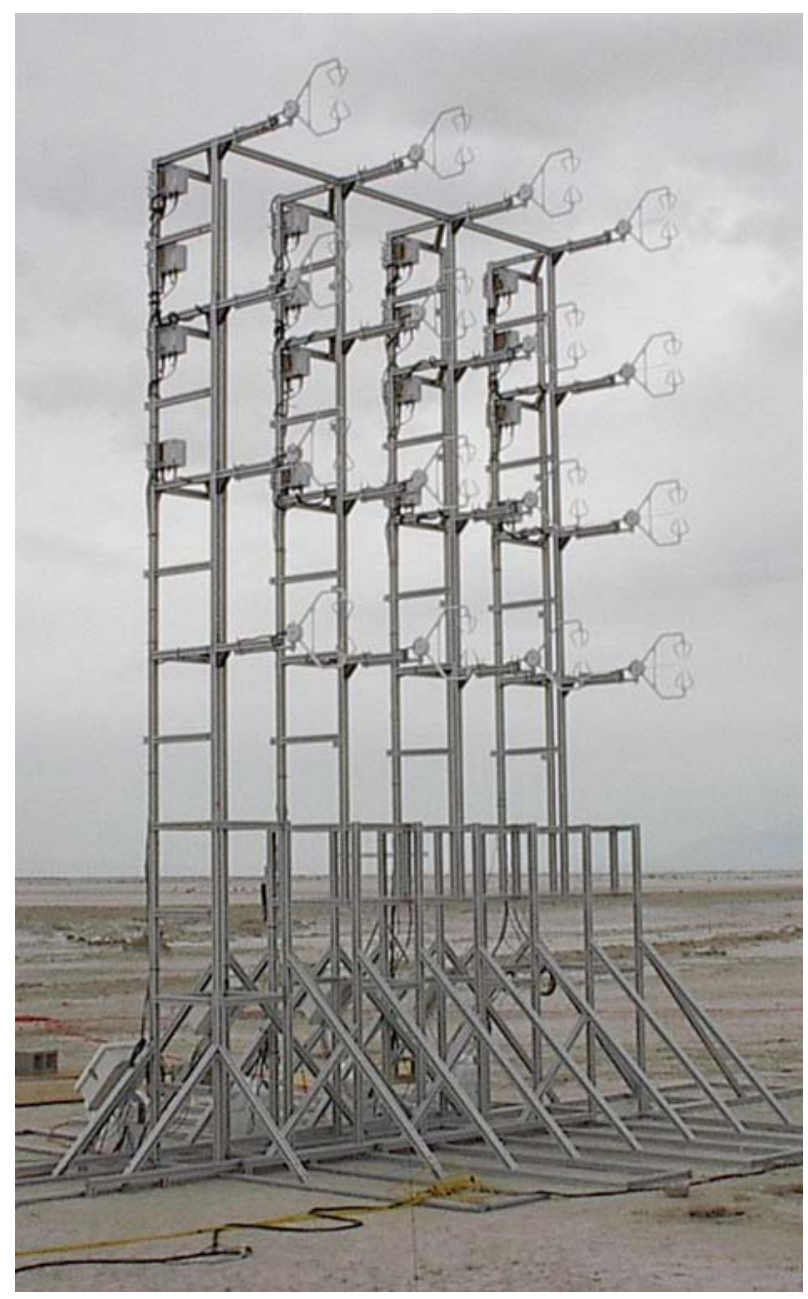

Fig. $14 \times 4$ sonic anemometer array used during the SGS2002 experiment, held in the western desert of Utah. 16 sonic anemometers were deployed in $4 \times 4$ grid allowing for the evaluation of derivatives required to study the SGS force and its parameterizations

the alignment parameter $\cos \theta$ and $\Pi_{*}=-\frac{\boldsymbol{\tau}_{i j} \tilde{S}_{i j}}{\left(\boldsymbol{\tau}_{p q} \tau_{p q}\right)^{\frac{1}{2}}\left(\tilde{S}_{m n} \tilde{S}_{m n}\right)^{\frac{1}{2}}}$ is computed, where $\Pi_{*}$ is the normalized SGS dissipation and is bound between -1 and 1 . This JPDF is presented in Fig. 4, and shows a bimodal structure. When the divergence of the $\boldsymbol{\tau}_{i j}$ obtained from the Smagorinsky model is aligned with the measured SGS force $(\cos \theta=1)$ there is a high likelihood that dissipation will be positive, $\Pi_{*}>0$. When the opposite is true $(\cos \theta=-1)$ there is a high likelihood that the dissipation will be negative, $\Pi_{*}<0$, indicating a backscatter of energy from small to large scales (Porté-Agel et al. 2001b). This result is not necessarily obvious since there is no direct relationship between the tensor contractions and the alignments of the divergence of the tensors. Yet, it is reassuring that the opposite alignments at the vector level yield consistent conclusions for the sign of the energy flux. 
Fig. 2 Probability density function showing the strong co-alignment between the SGS force and $\nabla \cdot \tau^{n l}$ calculated from the nonlinear model (solid line) and the bimodal alignment between the SGS force and $\nabla \cdot \boldsymbol{\tau}^{\text {Smag }}$ obtained from the Smagorinsky model (dashed line) under near-neutral stability

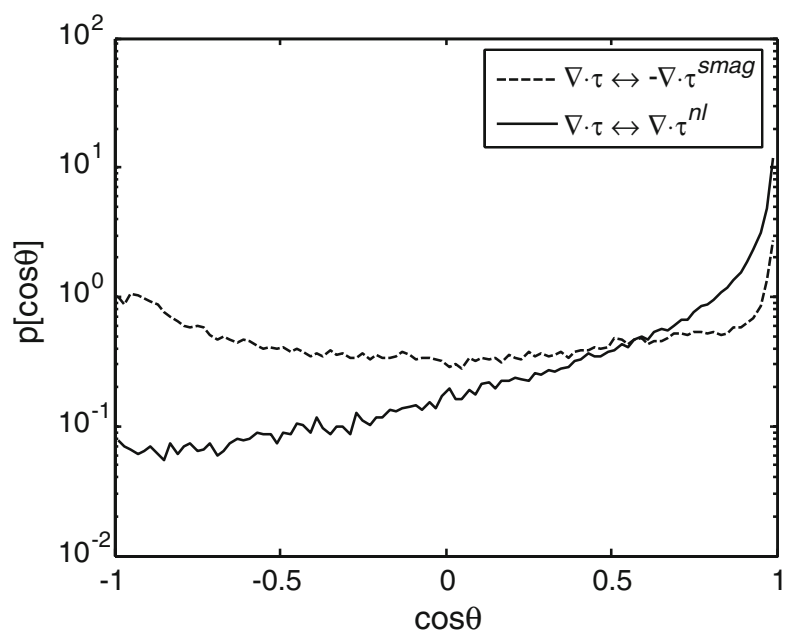

Fig. 3 PDF of alignment angle, including conditional sampling on SGS force magnitude. At high values of SGS force magnitude, the probability of co-alignment increases for both the divergence of the Smagorinsky model, $\nabla \cdot \tau^{\text {Smag }}$ and the divergence of the nonlinear model, $\nabla \cdot \tau^{n l}$ under near-neutral stability

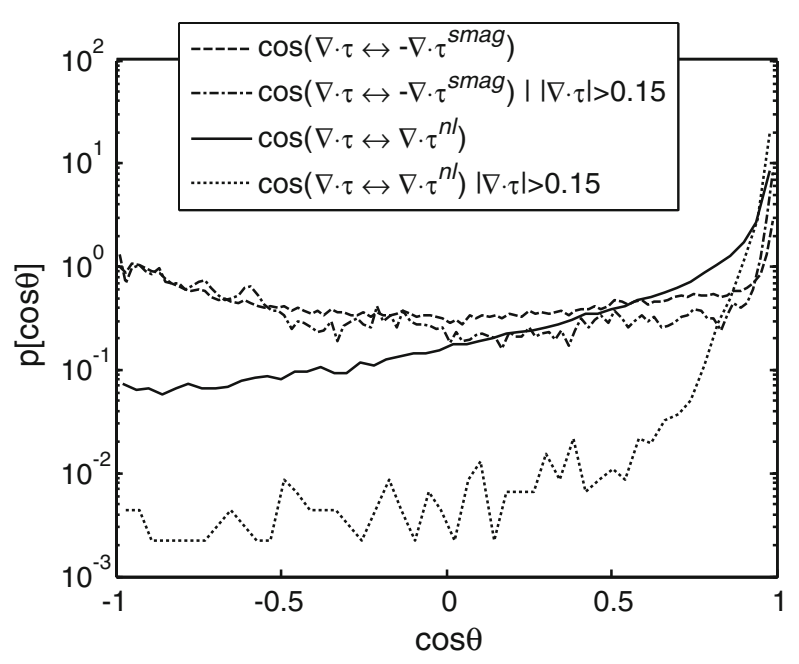

We now consider the effect of atmospheric stability on the alignment of the SGS forces. In Fig. 5 the PDFs previously shown (Figs. 2 and 3) are presented now for unstable and stable conditions. Recall that measurement periods with a value of $z / L<-0.1$ are classified as unstable, while segments with a value of $z / L>0.08$ are classified as stable. In Fig. 5a, PDFs of the cosine of the angle $\theta$ between measurements and models are plotted for unstable atmospheric conditions. The alignment of the SGS forces obtained from measurements and the nonlinear model is largely unchanged compared to that found under near-neutral stability. The alignment of the measured SGS force with the SGS force predicted by the Smagorinsky model shows increased probability of anti-alignment. In the case of stable atmospheric stability, Fig. 5b, the SGS force is still strongly aligned with $\nabla \cdot \tau^{n l}$, the divergence of the nonlinear model. However, the SGS force now has no preferential alignment with $\nabla \cdot \tau^{\text {Smag }}$, the divergence of the Smagorinsky model (indicated by the uniform PDF). This means that the alignment of the SGS force with $\nabla \cdot \tau^{\text {Smag }}$, the Smagorinsky model, is essentially random in the case of stable atmospheric conditions. 
Fig. 4 Joint probability density function of the normalized dissipation and the alignment between the SGS force and $\nabla \cdot \boldsymbol{\tau}^{\text {Smag }} \cdot$ Co-alignment corresponds to positive dissipation while anti-alignment corresponds to negative dissipation (backscatter) under near-neutral stability

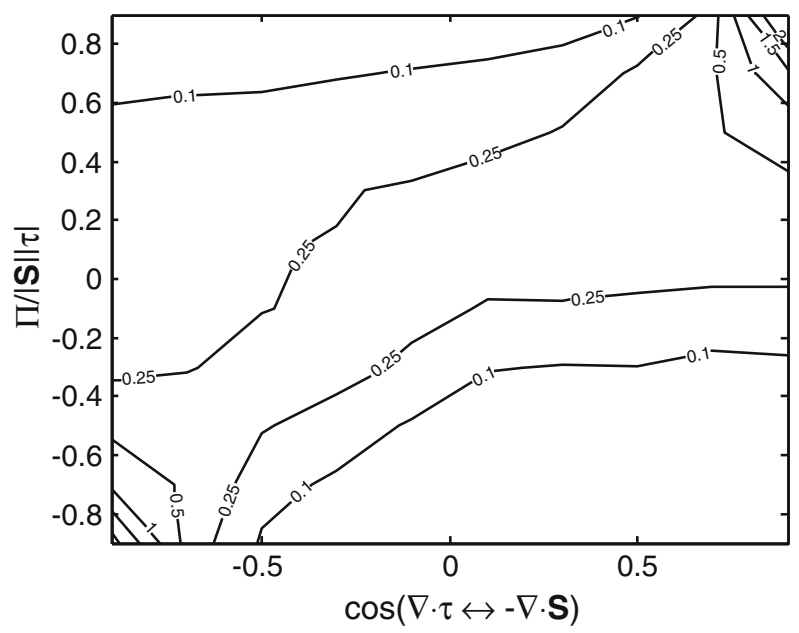

The joint alignments of the measured SGS force with the modelled SGS forces are presented by plotting the "mixed model span". The mixed model span is defined as the plane containing the two vectors $\nabla \cdot \tau^{\operatorname{Smag}}$ and $\nabla \cdot \tau^{n l}$. This analysis is similar to that presented in Higgins et al. (2004) who investigated SGS heat fluxes. The direction normal to the mixed model span is given by $\nabla \cdot \tau^{\text {Smag }} \times \nabla \cdot \tau^{n l}$. The projection, $\nabla \cdot \tau^{p}$, of the measured SGS force onto the plane spanned by the mixed model is the portion of the SGS force that can be expressed by the mixed model. Figure 6 shows the measured joint probability density function of $\theta\left[\nabla \cdot \tau^{\text {Smag }} \times \nabla \cdot \boldsymbol{\tau}^{n l} \leftrightarrow \nabla \cdot \boldsymbol{\tau}\right]$ and $\phi\left[\nabla \cdot \boldsymbol{\tau}^{p} \leftrightarrow \nabla \cdot \boldsymbol{\tau}^{n l}\right]$ plotted on the unit sphere. These joint PDFs quantify the relative frequency of the orientation of the measured SGS force relative to model-defined coordinates. In addition, a single PDF of $\zeta\left[\nabla \cdot \tau^{S m a g} \leftrightarrow \nabla \cdot \boldsymbol{\tau}^{n l}\right]$ shown at the bottom of Fig. 6 characterizes the alignment of the divergence of the filtered strain with respect to the divergence of the stress given by the nonlinear model. This analysis is presented for (a) unstable, (b) near-neutral, and (c) stable atmospheric stability. In all cases the measured SGS force is strongly aligned with the divergence of the stress given by the nonlinear model, however the relative position of the divergence of the stress given by the Smagorinsky model changes across atmospheric stabilities. In the case of unstable atmospheric stability (Fig. 6a), $\nabla \cdot \tau^{\text {Smag }}$ is both aligned and anti-aligned with the measured SGS force, $\nabla \cdot \tau$ which is consistent with the previous result seen in Fig. 5a. In the case of near-neutral atmospheric stability (see Fig. 6b), the measured and modelled forces are all co-aligned, indicating that the mixed model span is more likely to be a line, correctly orientated with the SGS force. Finally in the case of stable atmospheric conditions (see Fig. 6c), $\nabla \cdot \tau^{\text {Smag }}$ has a very week directionality mode perpendicular to both the SGS force and $\nabla \cdot \tau^{n l}$.

\section{Summary and Conclusions}

A field experiment was undertaken that allows the determination of the SGS force in the lower atmosphere based on spatial and temporal measurements of atmospheric turbulence. Analysis of the data shows that the SGS force obtained from the nonlinear model is strongly aligned with the measurements for all ranges of atmospheric stability. Conversely, the alignment of 
Fig. 5 PDFs of the alignment of the measured SGS force with the divergence of $\nabla \cdot \boldsymbol{\tau}^{\text {Smag }}$ (from the Smagorinsky model) and $\nabla \cdot \boldsymbol{\tau}^{n l}$ (from the nonlinear model) for a unstable, and $\mathbf{b}$ stable atmospheric conditions
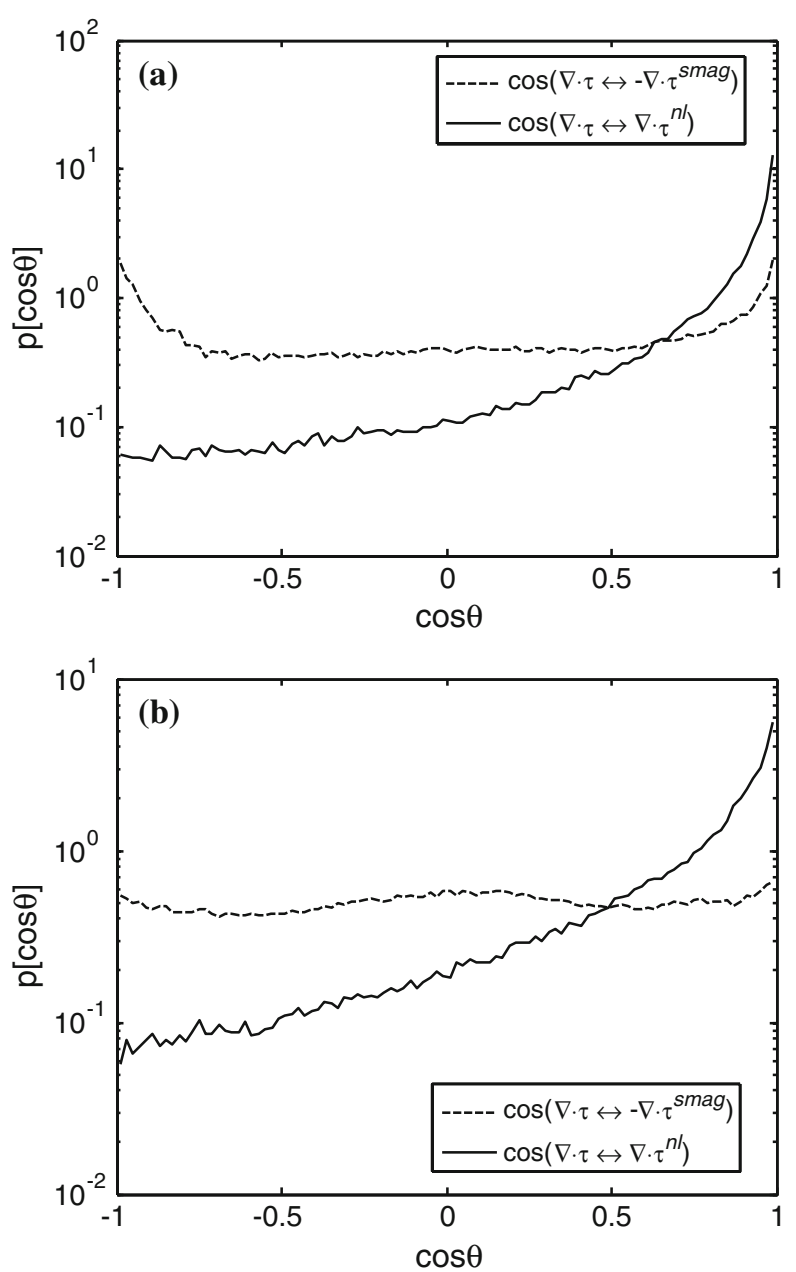

the SGS force with the force given by the divergence of the Smagorinsky model depends on atmospheric stability. In the case of near-neutral stability, $\nabla \cdot \tau^{\text {Smag }}$ is preferentially aligned with the SGS force, and has a weaker secondary anti-alignment. Under unstable atmospheric conditions, $\nabla \cdot \tau^{\text {Smag }}$ has nearly equal likelihood of co-alignment and anti-alignment with the measured SGS force. For stable atmospheric conditions, the alignment of the measured SGS force with $\nabla \cdot \tau^{\text {Smag }}$ is essentially "random". Analysis of joint PDFs confirms that anti-alignment of $\nabla \cdot \boldsymbol{\tau}$ with $\nabla \cdot \boldsymbol{\tau}^{\text {Smag }}$ was shown to coincide with energy backscatter events. Conditional sampling confirms the overall robustness of alignment trends with increasing magnitude of the measured SGS force, and reveals that there is an increased probability of co-alignment between the SGS force and models when the SGS force is large.

Analysis of the SGS force alignment relative to a 'mixed model span' reveals that this span is likely to be a line in the case of unstable and near-neutral atmospheric stability. In the stable case, a well-defined plane exists, but the SGS force is strongly aligned only with the SGS force given by the nonlinear model. 

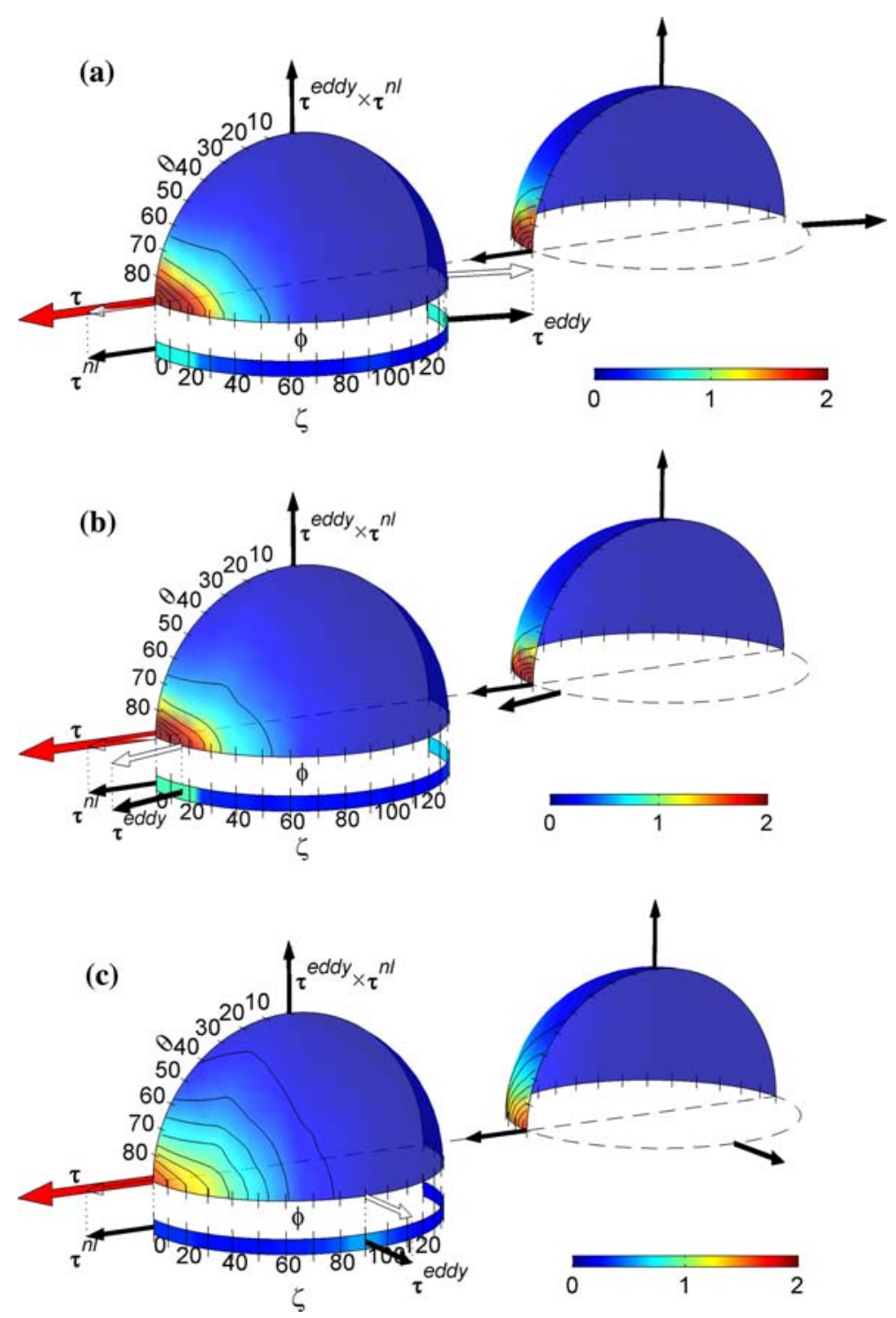

Fig. 6 Joint probability density functions showing the full alignment between the SGS force and the divergence of the filtered strain rate and the divergence of the nonlinear model for a unstable, $\mathbf{b}$ near-neutral, and $\mathbf{c}$ stable atmospheric conditions. In all cases the measured SGS force is preferentially aligned with $\nabla \cdot \tau^{n l}$, and the relative position of $\nabla \cdot \boldsymbol{\tau}^{\text {Smag }}$ varies for different stabilities

We note that SGS force considerations can play an interesting role in the dynamic determination of model parameters (Morinishi and Vasilyev 2002). Future studies to explore these ideas using field data would require additional sensors to be deployed since the dynamic model requires test-filtering at larger scales. 
Acknowledgements We gratefully acknowledge the field support and cooperation of Prof. Joe Klewicki who made the field campaign possible, and the support of colleagues Jan Kleissl, Vijayant Kumar, Fernando PortéAgel and colleagues from the Dugway Proving Ground. Partial funding was provided by (U.S.) NSF-ATM 0130766, NSF-EAR 0609690 and (Swiss) FNS 200021-109566.

\section{References}

Bardina J, Ferziger JH, Reynolds WC (1980) Improved subgrid scale models for large eddy simulation. American Institute of Aeronautics and Astronautics Paper 80-1357

Borue V, Orszag SA (1988) Local energy flux and subgrid-scale statistics in three-dimensional turbulence. J Fluid Mech 366:1-31. doi:10.1017/S0022112097008306

Clark RA, Ferziger JH, Reynolds WC (1979) Evaluation of subgrid-scale models using an accurately simulated turbulent flow. J Fluid Mech 91(1):1-16. doi:10.1017/S002211207900001X

Higgins CW, Parlange MB, Meneveau C (2003) Alignment trends of velocity gradients and subgrid-scale fluxes in the turbulent atmospheric boundary layer. Boundary-Layer Meteorol 109(1):59-83. doi:10. 1023/A: 1025484500899

Higgins CW, Parlange MB, Meneveau C (2004) The heat flux and the temperature gradient in the lower atmosphere. Geophys Res Lett 31(22):L22105. doi:10.1029/2004GL020053

Higgins CW, Parlange MB, Meneveau C (2007) The effect of filter dimension on the subgrid-scale stress, heat flux, and tensor alignments in the atmospheric surface layer. J Atmos Ocean Technol 24(3):360-375. doi:10.1175/JTECH1991.1

Horst TW, Kleissl J, Lenschow DH, Meneveau C, Moeng C-H, Parlange MB, Sullivan PP, Weil JC (2004) HATS, Field observations to obtain spatially-filtered turbulence fields from transverse arrays of sonic anemometers in the atmospheric surface layer. J Atmos Sci 61(13):1566-1581. doi:10.1175/ 1520-0469(2004)061<1566:HFOTOS $>2.0 . \mathrm{CO} ; 2$

Kleissl J, Parlange MB, Meneveau C (2004) Field experimental study of dynamic Smagorinsky models in the atmospheric surface layer. J Atmos Sci 61(18):2296-2307. doi:10.1175/1520-0469(2004)061<2296: FESODS $>2.0 . \mathrm{CO} ; 2$

Liu S, Katz J, Meneveau C (1994) On the properties of similarity subgrid-scale models as deduced from measurements in a turbulent jet. J Fluid Mech 275:83. doi:10.1017/S0022112094002296

Leonard A (1974) Energy cascade in large-eddy simulations of turbulent fluid flows. Adv Geophys 18:237. doi:10.1016/S0065-2687(08)60464-1

Meneveau C, Katz J (2000) Scale invariance and turbulence models for large-eddy-simulation. Annu Rev Fluid Mech 319:353-385

Morinishi Y, Vasilyev OV (2002) Vector level identity for dynamic subgrid scale modeling in large eddy simulation. Phys Fluids 14:3616-3623. doi:10.1063/1.1504450

Porté-Agel F, Meneveau C, Parlange MB (1998) Some basic properties of the surrogate subgrid-scale heat flux in the atmospheric boundary layer. Boundary-Layer Meteorol 88(3):425-444. doi:10.1023/ A:1001521504466

Porté-Agel F, Parlange MB, Meneveau C, Eichinger WE, Pahlow M (2000) Subgrid-scale dissipation in the atmospheric surface layer: effects of stability and filter dimension. J Hydrometeorol 1:75-87. doi:10. 1175/1525-7541(2000)001<0075:SSDITA > 2.0.CO;2

Porté-Agel F, Parlange MB, Meneveau C, Eichinger WE (2001a) A priori field study of the subgrid-scale heat fluxes and dissipation in the atmospheric surface layer. J Atmos Sci 58(18):2673-2697. doi:10.1175/ 1520-0469(2001)058<2673:APFSOT>2.0.CO;2

Porté-Agel F, Pahlow M, Meneveau C, Parlange MB (2001b) Atmospheric stability effect on subgrid scale physics for large-eddy simulation. Adv Water Resour 24(9-10):1085-1102. doi:10.1016/ S0309-1708(01)00039-2

Smagorinsky J (1963) General circulation experiments with primitive equations. Mon Weather Rev 91:99-164. doi:10.1175/1520-0493(1963)091<0099:GCEWTP>2.3.CO;2

Tong CN, Wyngaard JC, Brasseur JG (1998) Experimental study of the subgrid-scale stress in the atmospheric surface layer. J Atmos Sci 56(14):2277-2292. doi:10.1175/1520-0469(1999)056<2277:ESOTSS>2.0. $\mathrm{CO} ; 2$ 